\title{
die Hausarztpraxis
}

\section{Sleep Disorders: Guidance for the General Practitioner}

\author{
Patrick Heeb ${ }^{1}$ und René Fiechter ${ }^{2}$ \\ ${ }^{1}$ Lungenfachzentrum Fiechter, Aathal-Seegräben \\ ${ }^{2}$ Pneumologie/Somnologie Lungenfachzentrum Aathal-Seegräben, Schlafmedizin, Seeklinik Brunnen, Spital Einsiedeln
}

\section{Einleitung}

Schlafstörungen mit einer Prävalenz um $10 \%$ je nach epidemiologischer Methode haben einen enormen Einfluss auf die psychische und physische Gesundheit sowie die Lebensqualität, und durch Arbeitsausfälle oder Unfälle im häuslichen Umfeld und Strassenverkehr auch relevante sozioökonomische Implikationen. Eine Insomnie allein erhöht das Risiko, an einer Depression zu erkranken, wesentlich [1], und die obstruktive Schlafapnoe ist führend mit einer Prävalenz um 10-50 \%, je nach Cut-off-Wert [2]. Bereits die allgemeinärztliche Sprechstunde kann mit einer gezielten Anamnese und standardisierten Fragebögen (Scores) die Differenzialdiagnose eingrenzen und die Patientinnen und Patienten falls notwendig einer spezialisierten Diagnostik und Behandlung zuweisen.

Abbildung 1 verbildlicht den multidisziplinären Aspekt der Schlafstörungen, und so erhält die hausärztliche Sprechstunde eine essenzielle Steuerungsfunktion, um Schlafstörungen fach- und zeitgerecht zu erfassen. Hier können bereits erste Impulse in der Selbsthilfe und der adäquate Umgang mit Schlaf und Schlafhygiene vermittelt und im Bedarfsfall eine spezifische/apparative Diagnostik (zertifiziertes Schlaflabor/Somnologin/Somnologe) durchgeführt werden, um eine Chronifizierung der Schlafstörung oder einen Behandlungsaufschub einer somatischen Erkrankung zu verhindern. Im elektronischen Supplement (ESM) 1 sind einige zentrale Begriffe aus der Schlafmedizin kurz erläutert.

\section{Management von Personen mit Schlafstörungen}

\section{Anamneseerhebung}

Neben der Anamnese (interdisziplinäre Aspekte und Ko-Morbiditäten sind zu beachten: z.B. HNO-ärztliche, kardiologische, internistische, pneumologische, endokrinologische, psychiatrische) mit Erhebung auch der Tagesbefindlichkeit ist ebenso die Fremdanamnese essenziell [1]. Ein Fragebogen kann orientieren, wobei erst das persönliche Gespräch ein realitätsnahes Bild vermittelt, Missverständnisse ausschliesst und eine oft grobe Zuordnung der Schlafstörung schon erlaubt. Bereits die Unterscheidung in Tagesschläfrigkeit und Tagesmüdigkeit kann die Weichen stellen für die weitere Diagnostik und die spätere Zuordnung. So kann die Tagesschläfrigkeit subjektiv mit dem ESS-Fragebogen (Epworth Sleepiness Scale: 0-24 Punkte) quantifiziert werden: $>10 / 24$ Punkte dokumentieren eine abnorme Tagesschläfrigkeit. Die objektive Messung erfolgt im Schlaflabor mittels MSLT (Multiple Sleep Latency Test) oder MWT (Maintenance of Wakefulness Test/Multipler Wachbleibe-Test). Die Tagesmüdigkeit («Energielosigkeit») betrifft $20-30 \%$ der Hausarztpatientinnen und -patienten, dennoch fehlt eine objektive Messmethode, wobei der FSS (Fatigue Severity Scale: 1-7 Punkte) helfen kann.

Nach organischen Erkrankungen soll gezielt gefragt werden, z.B.:

Chronische Nierenerkrankungen, Magen-Darm-Erkrankungen, chronische Schmerz- oder rheumatologische Erkrankungen, endokrinologische Erkrankungen, Epilepsien, extrapyramidalmotorische Erkrankungen, Herz- und Lungenerkrankungen, Kopfschmerzsyndrome, maligne Erkrankungen, Polyneuropathien, Schlaganfall in der Anamnese, Multiple Sklerose, Juckreiz und Hauterkrankungen.

\section{Tools: Fragebogen, Scores}

Ein Anamnese-Fragebogen kann vorab der Patientin/dem Patienten ausgehändigt werden und enthält unter anderem Fragen zum Leitsymptom, zum Schlaf-Wach-Verhalten, Schlafzeiten, die Symptomabfrage der wichtigsten Schlafstörungen und Verdachtsdiagnosen und soll bereits bekannte somatische und psychische Erkrankungen erfassen. 
Im ESM 2 steht unser Fragebogen als Beispiel der Symptomabfrage im Rahmen der Schlafanamnese als ExcelDokument zur Verfügung und kann den eigenen Bedürfnissen angepasst werden (adaptiert nach R. Fiechter [3]).

\section{Medikamente}

Schlafstörungen können durch Medikamente, Drogen oder Substanzen verursacht oder begünstigt werden. Durch direkte Wirkung oder Interaktion kann die Homöostase der Schlaf-Wachregulation destabilisiert werden [4]. Ganz allgemein können Antidepressiva, Neuroleptika oder Psychopharmaka sowohl Schläfrigkeit, Schlafstörungen als auch Wachheit verursachen. Im Speziellen die Schläfrigkeit kann ausgelöst oder begünstigt werden durch z.B. Opioide, kaliumsparende Diuretika, Anticholinergika, Methyldopa, Antiepileptika, Parkinsonmittel; Schlafstörungen z.B. durch Glukokortikoide, Betarezeptorenblocker oder NSAR.

\section{Fahreignung}

Bei Vorliegen einer Tagesschläfrigkeit muss die Fahreignung beurteilt, mit der Patientin/dem Patienten besprochen und auch in der Krankenakte dokumentiert werden. Da das Ausmass der Tagesschläfrigkeit nicht gut mit den objektivierten Befunden oder den Diagnosen korreliert, ist in der Beurteilung der Fahreignung entscheidend, inwiefern die/der Betroffene die Schläfrigkeit wahrnimmt und wie er damit umgeht [5]. Die Selbstverantwortung ist im Strassenverkehr das oberste Prinzip. Sofern ein Unfall in der Akte eines Berufs- oder PKW-Fahrers mit Tagesschläfrigkeit zu verzeichnen ist, ist die Abklärung in einem spezialisierten Schlafzentrum empfohlen. Gemäss Strassenverkehrsgesetz (SVG) sind alle Ärzte berechtigt (nicht verpflichtet), eine Meldung bei der Strassenverkehrsbehörde zu tätigen, wenn die Fahreignung angezweifelt wird.

\begin{tabular}{|c|c|c|}
\hline \multicolumn{3}{|c|}{ Grunderkrankung oder Ko-Morbidität } \\
\hline $\begin{array}{l}\text { Internistisch } \\
\text { Chronische Nierenerkrankungen. } \\
\text { Magen-Darm-Erkrankungen (z.B. } \\
\text { Leberinsuffizienz). } \\
\text { Chronische Schmerzen. Somatoforme Störung. } \\
\text { Rheumatologische Erkrankungen. } \\
\text { Endokrinologische Erkrankungen } \\
\text { (z.B. Hypothyreose). } \\
\text { Herzerkrankungen (z.B Herzinsuffizienz). } \\
\text { Lungenerkrankungen. Malignome. } \\
\text { Hauterkrankungen. Pruritus. }\end{array}$ & $\begin{array}{l}\text { Epilepsien. } \\
\text { Extrapyramidalmotorische Erkrankungen. } \\
\text { Kopfschmerzen. Polyneuropathien. } \\
\text { St. n. zerebrovaskulärem Insult. } \\
\text { Multiple Sklerose. } \\
\text { Neurodegenerative Erkrankungen. Demenz. } \\
\text { St. n. Schädelhirntrauma. } \\
\text { Neuromuskulär (z.B. Myasthenia gravis, Myotone } \\
\text { Dystrophie). }\end{array}$ & 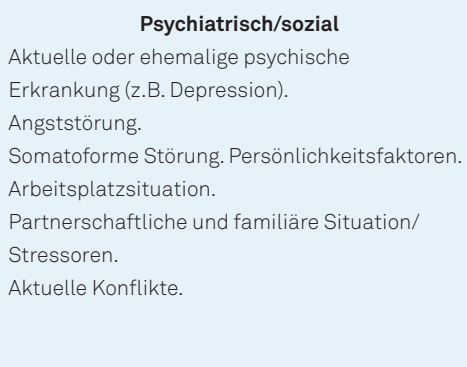 \\
\hline
\end{tabular}

Medikamente/Toxen/Substanzen

Alkohol. Rauschmittel. Antibiotika (z.B. Gyrasehemmer). Antidementiva (z.B. Piracetam). Antriebssteigernde Antidepressiva (z.B. SSRI). Blutdruckmittel (z.B. Betablocker). Antiasthmatika (z.B. Theophyllin, Betasympathomimetika). Diuretika. Hormonpräparate (z.B. Thyroxin, Steroide). Stimulierende Substanzen (z.B. Koffein, Nikotin, Amphetamin, Ecstasy).

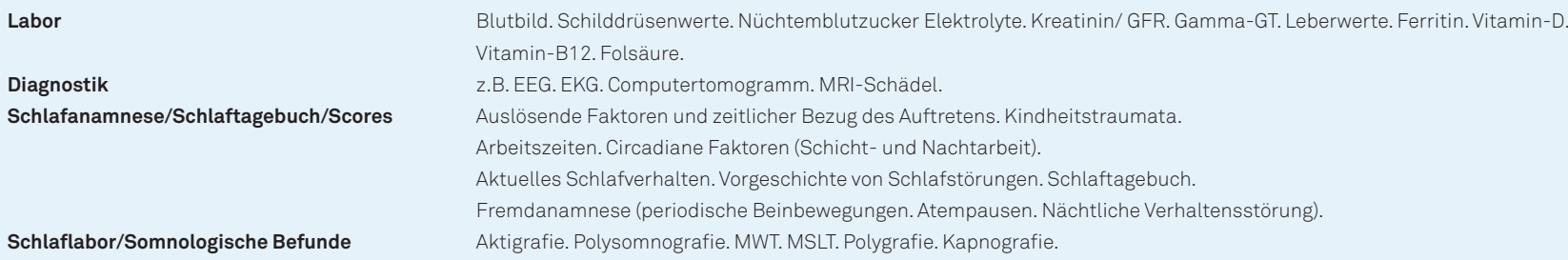

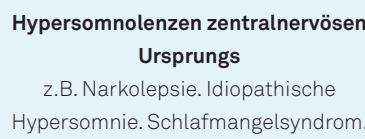

Schlafbezogene Atemstörung

z.B. OSAS. Cheyne-Stokes-Atmung. Hypoventilationssyndrome.
Zirkadiane Schlaf-Wach-

Rhytmusstörung

z.B. Schichtarbeit. Jetlag.
Parasomnien

z.B. NREM-/REM-Parasomnien. Nachtwandeln.
Schlafbezogene Bewegungsstörung

z.B. Restless-Legs-Syndrom

Bruxismus. 


\section{Mögliche initiale Schlaftherapie und Instruktion durch den Erstversorger}

Bereits durch den Erstversorger können allgemeine Informationen, die Schlafhygieneregeln und Strategien zur Prävention von Schlafstörungen vermittelt werden und allenfalls Anpassungen der Ruhezeiten an den zirkadianen Rhythmus sowie Entwöhnung oder Änderungen schlafstörender Substanzen oder Medikamente erfolgen. Auch die Behandlung von Ko-Morbiditäten kann relevante Auswirkungen auf den Schlaf haben. Die spezifische und schrittweise Insomnietherapie (z.B. Kognitive Verhaltenstherapie für Insomnie (KVT-I), Progressive Muskelrelaxation, Achtsamkeitsübungen etc.) sollte durch einen erfahrenen Therapeuten erfolgen.

Stichwortartig ist die Basisintervention (nach [6]) unten aufgeführt:

Regeln für einen gesunden Schlaf/Schlafhygiene

- Nach dem Mittagessen keine koffeinhaltigen Getränke (Kaffee, Schwarztee, Cola) mehr trinken

- Nach Möglichkeit Verzicht auf Alkohol, insbesondere nicht als Schlafmittel verwenden

- Vermeiden schwerer Mahlzeiten am Abend

- Regelmässige körperliche Aktivität

- Allmähliche Verringerung geistiger und körperlicher Anstrengung vor dem Zubettgehen

- Ein persönliches Einschlafritual einführen

- Angenehme Schlafzimmeratmosphäre schaffen (ruhig, verdunkelt, nicht zu warm)

- Nachts nicht auf Wecker oder Armbanduhr schauen

\section{Instruktionen zur Stimuluskontrolle}

- Erst ins Bett gehen, wenn man schläfrig ist

- Das Bett nur zum Schlafen nutzen, nicht zum Lesen, Trinken, Rauchen oder Fernsehen

- Wenn nach 15 Minuten der Schlaf nicht einkehrt, in ein anderes Zimmer gehen und erst erneut ins Bett gehen, wenn man schläfrig ist

- Konstante Aufstehzeit am Morgen

- Vermeiden von Tagesschlaf (bei Bedarf max. 30 Minuten)

\section{Bettzeitenrestriktion}

- Ermitteln der subjektiven Schlafzeit anhand eines 14-tägigen Schlaftagebuchs

- Setzen der Bettzeit auf die durchschnittliche Schlafzeit (Daten aus dem Schlaftagebuch), jedoch nicht weniger als 5 Stunden

- Re-Evaluation der Bettzeitenrestriktion: Bei einer Schlafeffizienz (Anteil effektiver Schlafzeit an der Bettzeit) grösser $85 \%$ kann die Schlafdauer um 30 Minuten verlängert werden

\section{Modifizierte Bettzeitenrestriktion}

\section{für die Hausarztpraxis (nach [6, 7])}

Führen eines 14-tägigen Schlaftagebuches. Ermittlung der durchschnittlichen Schlaf- und Bettzeit. Die Bettgehzeit wird errechnet, indem die Summe der durchschnittlichen Schlafzeit $+50 \%$ der Wachzeit im Bett, ausgehend von der gewünschten Aufstehzeit, rückgerechnet wird.

\section{Hypnotika (nach $[1,6])$}

Zwar sind Benzodiazepine und die neuen BenzodiazepinAgonisten (Z-Substanzen/z-Drugs) im Kurzzeitgebrauch etwa gleich effektiv wie die kognitive Verhaltenstherapie für Insomnie (KVT-I), ihr Einsatz aufgrund des Abhängigkeitspotenzials und der möglichen unerwünschten Nebenwirkungen ist aber problematisch, und eine Langzeitbehandlung sollte nicht erfolgen. Auch für sedierende Antidepressiva ist die Effektivität bewiesen, aber auch hier fehlt eine Empfehlung zur Langzeittherapie aufgrund möglicher Nebenwirkungen und Risiken. Für Antipsychotika fehlt eine Empfehlung in der Insomniebehandlung mit Ausnahme niedrigpotenter Antipsychotika bei gerontopsychiatrischen Patientinnen und Patienten. Prinzipiell wird auch das Melatonin aufgrund geringer Wirksamkeit in der Insomniebehandlung nicht generell empfohlen. Für Baldrian und Phytopharmaka ist die aktuelle Datenlage unzureichend. Die möglichen Pharmaka-Nebenwirkungen sollten geprüft werden, zum Beispiel mittels Konsultation der Medikamentenkompendien (z.B. www.swissmedicinfo.ch).

\section{Klassifikation der Schlafstörungen in Anlehnung an die ICSD-3 [8]}

Die ICD-10 hat zwar ihren Stellenwert in der Dokumentation und im Abrechnungswesen, ist aber aus wissenschaftlicher Sicht im Vergleich zur ICSD-3 und den S3-Leitlinien veraltet, zumal über die letzten drei Jahrzehnte die Schlafmedizin wesentliche Fortschritte erzielt hat. Da die DSM5- und auch die S3-Leitlinien sich an der ICSD-3 orientieren und die ICD-11 erst 2022 offiziell erscheinen wird, orientieren sich die Schlafmediziner meist an den sieben Kategorien der Schlafstörungen nach ICSD-3.

\section{Vignetten wichtiger Schlafstörungen (adaptiert nach ICSD-3 $[1,4]$ )}

\section{Insomnie}

Charakterisiert durch Ein- oder Durchschlafstörung, Früherwachen mit Beeinträchtigung der Schlafqualität und Tagesbefindlichkeit, mindestens dreimalig pro Woche, bei der chronischen Insomnie über mindestens drei Monate 
bestehend. Bis zu 30\% der Erwachsenen geben mindestens ein Symptom der Insomnie an, und die Insomnie-Kriterien erfüllen zirka $10 \%$.

Klinische Fragen: Die Anamnese kann sehr umfassend sein mit Erfragung der Art der Schlafstörung (Einschlafen, Durchschlafen, Früherwachen, nichterholsamer Schlaf, Art und Dauer der Probleme), der Schlafhygienemassnahmen (Störfaktoren im Schlafzimmer, unregelmässiger Schlaf-Wach-Rhythmus, Bettzeiten, Schlafdauer, Verhalten bei nächtlichem Erwachen), von Auffälligkeiten in den Lebensumständen (Stress, Konflikte), der Ko-Morbiditäten (z.B. Restless Legs, Schmerzen, Dyspnoe, internistische/neurologische/psychiatrische Erkrankung, Nykturie, Schlafapnoe), der Medikamente, Substanzen oder Stimulanzien (z.B. Theophyllin, Betasympathomimetika, Diuretika, Hormonpräparate, Koffein, Nikotin, Amphetamin, Ecstasy), welche eine Insomnie begünstigen können.

Diagnostik: Anamnese mit Fragebögen. Wenn die Schlafstörung über Wochen ohne klaren Grund persistiert, sollte die Zuweisung zur Somnologin/zum Somnologen/ zum Schlaflabor erwogen werden.

Therapie: Je nach Erfahrung oder Ausbildung kann bereits eine Schlafberatung durch den Erstversorger zur Verbesserung der Schlafhygiene (siehe Management von Personen mit Schlafstörung) beitragen. Alternativ können durch geschulte Spezialisten/Insomnietherapeuten die erprobten Therapien in mehreren Sitzungen erlernt und umgesetzt werden. Dabei kommen zum Beispiel kognitivverhaltenstherapeutische Verfahren, Psychoedukation, progressive Muskelrelaxation nach Jacobson, verhaltenstherapeutische Massnahmen (z.B. Bettzeitenrestriktion, Stimuluskontrolle) und weitere Therapieformen (z.B. Entspannungsübungen, autogenes Training, Achtsamkeitstraining, Paradoxe Intervention, Selbsthypnose/Hypnose) zur Anwendung.

\section{Schlafbezogene Atemstörung, exemplarisch die obstruktive Schlafapnoe}

Eingeteilt in die obstruktive und zentrale Schlafapnoe (z.B. zentral, gemischt, komplex, Cheyne-Stokes-Atmung etc.) sowie die Hypoventilationssyndrome (z.B. Adipositashypoventilation), gehören die schlafbezogenen Atemstörungen je nach gewählten Kriterien zu den prävalentesten Schlafstörungen mit enger Assoziation zu kardiovaskulären Erkrankungen. Eine adäquate Therapie trägt zu einer signifikanten Risikoreduktion für kardiovaskulären Ereignisse und zur Verminderung der Unfallgefahr bei.

Klinische Fragen: Klassische Symptome (Schnarchen, beobachtete Apnoen, nächtliches Choking, Mundtrockenheit, dumpfer morgendlicher Kopfschmerz, Abgeschlagenheit, Leistungsabfall, Nykturie, Wesensveränderung, Reizbarkeit, hoher Blutdruck, Herzrhythmusstörungen, Adipositas, Diabetes mellitus, Libidoverlust, Potenzstörungen) können auf eine Schlafapnoe/-hypopnoe hinweisen.
Diagnostik: Die zusätzlichen Scores für exzessive Tagesschläfrigkeit (z.B. NoSAS $\geq 9 / 17$ Punkte, ESS $>10 / 24$ Punkte, STOP-BANG) können die Vortestwahrscheinlichkeit für das Vorliegen einer Schlafapnoe erhöhen. Bei hoher Vortestwahrscheinlichkeit kann auf eine respiratorische Polygrafie zurückgegriffen werden, ansonsten ist die Polysomnografie der diagnostische GoldStandard, um andere somnologische Erkrankungen, welche die Symptomatik auch erklären könnten, nicht zu verpassen. Um der hohen Prävalenz der schlafbezogenen Atemstörungen zu genügen, wurden vereinfachte diagnostische Verfahren (1-3-kanalige Systeme, z.B. Pulsoxymetrie, ApneaLink) entwickelt, welche bei wenig Symptomatischen oder Lungengesunden jedoch Limitationen in der Sensitivität und Spezifität aufweisen $[1,4]$. Die Zusammenarbeit mit einem Schlafmediziner oder einem schlafmedizinischen Zentrum kann Unklarheiten beseitigen oder weiterführende standardisierte Prozesse (weitere Diagnostik, Therapie, Beatmung) sicherstellen. Die Frage nach der Fahreignung muss ebenfalls evaluiert werden.

Therapie: Die etablierteste Therapie ist die kontinuierliche Überdrucktherapie (CPAP) mit Verbesserung der Tagesschläfrigkeit, der Lebensqualität und der Tagesvigilanz [1]. Flankierende Massnahmen stellen diätetische Massnahmen/Gewichtsreduktion und Einhaltung der Schlafhygienemassnahmen dar. Die BPAP- und ASVHeimbeatmung kommt bei OSAS mit Hypoventilation und zentralen Atemstörungen zum Einsatz. Alternativen sind je nach Situation oder Verträglichkeit die Unterkieferprotrusionsschiene und Lagetherapie. Für das Muskeltonustraining (Didgeridoo, transkutane Elektrostimulation) fehlen Langzeiterfahrungen. Die Hypoglossusstimulation und operative Methoden sollen nur bei einem streng selektionierten Patientenklientel angewandt werden. Neueste pathophysiologische Arbeiten stellen zudem die rein mechanischen Erklärungsversuche in Frage (Atemantrieb, Reaktionsbereitschaft der Muskulatur, Neigung zu Weckreaktionen etc.), und so bleibt die Hoffnung, dass weitere Therapieformen neben den mechanischen in Zukunft einen grösseren Stellenwert bekommen.

\section{Restless-Legs-Syndrom}

Klinische Fragen: Anamnestisch kann die Diagnose des Restless-Legs-Syndroms anhand pathognomonischer Symptome gestellt werden [9]. Gefordert sind der Bewegungsdrang mit/ohne Missempfindung der Beine (selten auch Arme) mit Aggravation in Ruhe (bevorzugt abends oder nachts), gemildert durch Umhergehen und fehlende anderweitige Erklärung (z.B. Myalgie, chronisch venöse Insuffizienz, Beinödeme, Arthritis, Beinkrämpfe). Unterstützt wird die Diagnose bei Ansprechen auf dopaminerge Behandlung (L-Dopa-Test), positiver Familienanamnese oder bei Vorliegen einer periodischen Beinbewegung in der Polysomnografie (PLM). Assoziiert ist das RLS mit 
Schwangerschaft, Medikamenten (z.B. Quetiapin, Citalopram, Mirtazapin, Östrogene, Simvastatin), Eisenmangel, Urämie, Diabetes mellitus, Rheumatoider Arthritis oder Parkinson.

Diagnostik: Die neurologische Untersuchung ist unauffällig und der IRLS-Score erreicht mindestens 15/40 Punkte. Laborchemischer Ausschluss von Ko-Faktoren (z.B. Ferritin, Blutbild, Nieren-, Entzündungs- und Schilddrüsenwerte, Blutzucker). Beim Spezialisten die Polysomnografie, Elektroneurografie, Aktimetrie oder der L-Dopa-Test.

Therapie: Neben allgemeinen Hinweisen (Vermeidung von Alkohol, Koffein und RLS-auslösenden Medikamenten, der Therapie von Begleiterkrankungen und unterstützend Sport) werden dopaminerge und weitere Substanzen eingesetzt (Therapieempfehlung bei neurologischer/somnologischer Praxis einholen). Auch eine Eisensubstitution im Falle eines Ferritinwertes $<50 \mathrm{mcg} / \mathrm{l}$ ist empfohlen.

\section{Insufficient Sleep Syndrome (Schlaf- insuffizienzsyndrom, verhaltensinduziertes Schlafmangelsyndrom, Schlafmanko)}

Klinische Fragen: Leitsymptom ist die Tagesschläfrigkeit und/oder Erhöhung des Schlafbedürfnisses (Hypersomnie) durch das verhaltensinduzierte Schlafmanko. Wichtig in der Diagnosestellung ist die Anamnese (Schlafdauer an Arbeitstagen und freien Tagen) mit Verbesserung der Tagesschläfrigkeit nach Ausschlafen (z.B. Urlaub, Wochenende) oder verlängerter Schlafdauer.

Diagnostik: Die Aktigrafie mit Führen eines Schlaftagebuchs/Schlaf-Wach-Protokolls kann in der Regel von weiteren ZNS-Hypersomnien (z.B. der idiopathischen Hypersomnie) unterscheiden, wobei ein unterschiedlicher Schlafbedarf bei den Patientinnen und Patienten beachtet werden muss. Die Verlängerung der Schlafdauer kann diagnostisch und therapeutisch sein.

Die Diagnostik der weiteren ZNS-Hypersomnien ist diagnostisch aufwändiger. Erfasst werden die Begleitsymptome, der klinische Kontext und die bereits erfolgten Abklärungen. Je nach Verdachtsdiagnose müssen ergänzende Untersuchungen erwogen werden (z.B. Video-Polysomnographie, Vigilanztests (z.B. Multipler Schlaflatenztest), Bildgebung (z.B. MRI-Schädel), Liquor- und Laboruntersuchungen sowie fachübergreifende ergänzende Diagnostik (z.B. neurologisch, internistisch, psychiatrisch).

Therapie: Einhalten der Schlafhygiene und individuellen Schlafdauer.

\section{Idiopathische Hypersomnie}

Klinische Fragen: Subjektive Tagesschläfrigkeit und Schlaftrunkenheit. Verlängerte Schlafdauer oder TagesNaps verbessern die Tagesschläfrigkeit nicht [1].

Diagnostik: Die idiopathische Hypersomnie eine Ausschlussdiagnose mit verkürzter Schlaflatenz im MSLT $(<8$ Min.) oder $>660$ Minuten/Tag durchschnittlicher Schlaf- dauer in der Aktigrafie, weniger als 2 SOREM in der PSG, Fehlen von Kataplexie und Hypokretinmangel.

Therapie: Nichtmedikamentöse Therapien (Verhaltensmassnahmen), welche ansonsten essenziell in der Behandlung der Tagesschläfrigkeit und Hypersomnie sind, greifen bei der Idiopathischen Hypersomnie kaum. Dennoch sollten die Massnahmen der Schlafhygiene vermittelt werden und ggf. Tages-Naps versucht werden. Trotz fehlender offizieller Medikamentenzulassung besteht für Modafinil die beste Evidenz. Auch Methylphenidat wird von Somnologinnen und Somnologen eingesetzt und kann die Tagesschläfrigkeit verbessern und die Einschlaflatenz in monotonen Situationen verlängern, obwohl eine Normalisierung in der Regel nicht erreicht wird. Therapieresistenz und Nebenwirkungen sind nicht selten, und die Eskalation auf Amphetamine muss sehr sorgfältig (Suchtpotenzial, Blutdruckerhöhung, Verschlechterung des kardiovaskulären Risikos) geprüft werden. Weitere medikamentöse Optionen ohne bisherige Evidenz werden in spezialisierten neurologischen und schlafmedizinischen Zentren geprüft.

\section{Schlafstörung durch Medikamente}

Die Beeinflussung des Schlafs (z.B. Hypersomnie, Parasomnie, nächtliche Bewegungsstörung, schlafbezogene Atemstörung) aufgrund von Medikation oder Substanzen kann vielfältig sein. Zum Beispiel können Antidepressiva/ Betablocker/Alkoholentzug eine REM-Verhaltensstörung, das Zolpidem/Antipsychotika/Antidepressiva/Ciprofloxacin eine Non-REM-Parasomnie und Antidepressiva generell ein Restless-Legs-Syndrom auslösen oder verstärken. Die medikamentöse Triggerung z.B. einer latenten REMVerhaltensstörung kann eine beginnende neurodegenerative Erkrankung frühzeitig demaskieren [1].

Klinische Fragen: Ein zeitlicher Zusammenhang zur Einnahme von Medikamenten/Substanzen ist vorhanden.

Diagnostik: Erfassen der Genussmittel, Medikamente, Schlafmittel, Stimulanzien und Substanzen.

Therapie: Anpassung der Medikation, Dosisreduktionsversuch, Absetzversuch, Information zum Substanzgebrauch. Insbesondere Hypnotika sollen kritisch geprüft werden; eine Anwendung für mehr als einige Wochen ist nicht empfohlen. Erwägung einer ambulanten/stationären Insomnietherapie.

\section{Schlafstörung durch Systemerkrankungen}

Klinische Fragen: Erfassung insbesondere internistischer, neurologischer und psychiatrischer Ko-Morbiditäten. Gegenseitige Korrelationen bestehen insbesondere für kardiovaskuläre Erkrankungen.

Diagnostik: Die Diagnostik richtet sich nach den Verdachtsdiagnosen und im Wissen um eine gegenseitige Korrelation insbesondere für kardiovaskuläre Erkrankungen und Schlafstörungen. So ist die Schlafapnoe selbst 
ein Risikofaktor für kardiovaskuläre Erkrankungen und umgekehrt sind z.B. die Herzinsuffizienz und das Vorhofflimmern ein möglicher Auslöser einer zentralen Atemstörung. Die COPD kann ein hyperkapnisches Atemversagen bedingen und eine nichtinvasive Beatmung notwendig machen. Auch chronische Schmerzen können durch die Behandlung einer Insomnie/Schlafapnoe gebessert werden. Psychiatrisch Belastete oder Tumorkranke erfahren zu einem hohen Prozentsatz eine Fatigue (Ausschlussdiagnose) oder Insomnie.

Therapie: Die Therapie richtet sich nach den Grunderkrankungen und den ergänzenden Therapien der Schlafmedizin.

\section{Schlafstörung durch psychiatrische Erkrankungen}

Klinische Fragen: Ein- und Durchschlafstörungen und Gedankenkreisen als Korrelat einer Insomnie werden bei einem Grossteil der depressiven Patientinnen und Patienten beschrieben.

Diagnostik: Polysomnografisch objektivierte Veränderungen in der Schlafkontinuität, der Schlafstadienverteilung und REM-Charakterisierung können umgekehrt differenzialdiagnostische Hinweise für eine psychiatrische Erkrankung liefern. Die Evaluation des Schlafs soll Bestandteil der psychischen Therapieplanung sein. Schlafstörungen können ein Frühsymptom einer psychischen Erkrankung sein, aber auch nach deren Behandlung per- sistieren. Organisch bedingte Schlafstörungen können als Ko-Morbidität vorhanden sein und machen ggf. eine technische Diagnostik notwendig (Aktigrafie, Polysomnografie, Polygrafie, Labordiagnostik).

Therapie: Unter psychiatrischer Behandlung bessern sich oft die Schlafstörungen. Bei Persistenz sollte eine spezifisch-somnologische Therapie (z.B. Kognitive Verhaltenstherapie für Insomnie (KVT-I) erfolgen, die den Verlauf der psychiatrischen Erkrankung ebenfalls positiv beeinflussen kann.

\section{Spezifische Diagnostik, Schlaflabor}

Kann die Schlafstörung nicht adäquat eingeordnet oder behandelt werden, ist die Zuweisung an eine Somnologin/ einen Somnologen oder ein zertifiziertes Schlaflabor empfohlen mit der Möglichkeit der apparativen Untersuchung (z.B. Polygrafie, Aktigrafie, Video-Polysomnografie, Kapnografie, MWT, MSLT). Die Abbildung 2 bietet eine grobe Übersicht der Wechselbeziehungen an Symptomen, Scores, möglicher Diagnosen, spezifischer Diagnostik und möglicher Therapien, welche in die Überlegungen des Somnologen einfliessen. Indikationen für die polysomnografische Untersuchung sind trotz Kostendruck weiterhin die therapieresistente Insomnie, der Verdacht auf eine organisch bedingte Insomnie, Schlafapnoe oder periodische Beinbewegungen, des Weiteren bei gutachterlichen Fragen, im Rahmen der Forschung oder der Insomnie-Abklä-

\footnotetext{
MÖGLICHE SYMPTOME

- Einschlafstörung

- Durchschlafstörung

- Früherwachen

- Nichterholsamer Schlaf

- Tagesmüdigkeit

- Tagesschläfrigkeit

- Schnarchen

- Apnoen

- Mundtrockenheit

- Dumpfer morgendlicher

Kopfschmerz

- Abgeschlagenheit

- Leistungsabfal

-Wesensveränderung

- Reizbarkeit

- Hoher Blutdruck

- Herzrhythmusstörung

- Adipositas

- Diabetes mellitus

- Libidoverlust

• Potenzstörung
}

EVALUTATION/DIAGNOSTIK

- Schlafanamnese/Medikamenten-

und Substanzanamnese

- Erfassung Ko-Morbiditäten

- Labor/Bildgebung

- Schlaftagebuch/Schlaf/

Wach-Protokoll

- Anpassung Schlafdauer

(z.B. bei Schlafmangelsyndrom)

- Restless-Legs-Anamnese (IRLS)

- Epworth Sleepiness Scale

(ESS) $>10 / 24$ Punkte

- NoSAS2: $\geq 9 / 17$ Punkte

STOP-BANG

- Fatigue Severity Scale

(FSS) > 4/7 Punkte

- Pulsoxymetrie/Apnealink

\section{SPEZIFISCHE DIAGNOSTIK SCHLAFLABOR/ SOMNOLOGE}

- Polygrafie

- Aktigrafie

- Polysomnografie

- Kapnografie

- Labor (z.B. HLA)/Liquor

(z.B. Hypokretin)

- Vigilanztests

- MWT/MSLT

- Fahreignungsprüfung

- Internistische, neurologische,

psychiatrische Abklärung je nach

Verdachtsdiagnose

\author{
SCHLAFSTÖRUNG \\ - Insomnie \\ - Schlafbezogene Atemstörung \\ - Schlafstörung durch \\ Medikamente, Substanzen \\ - Narkolepsie \\ - Schlafmangelsyndrom \\ - Restless-Legs-Syndrom \\ - Idiopathische Hypersomnie \\ - Schlafstörung durch \\ Systemerkrankung \\ - Schlafstörung durch \\ Medikamente \\ - Schlafstörungen bei \\ internistischen/neurologischen/ \\ psychiatrischen Erkrankungen
}

Vermitteln der Schlafhygienemassnahmen/Stimuluskontrolle/Anpassung der Ruhezeiten an den zirkadianen Rhythmus/Bettzeitenrestriktion, Entwöhnung, Umstellung, Dosisreduktion von schlafstörenden Medikamenten/Substanzen, Behandlung von Ko-Morbiditäten, Kognitive Verhaltenstherapie für Insomnie (KVT-1), CPAP/Heimventilation (z.B. Bilevel, ASV)/Unterkiefer-Protrusionsschiene/Gewichtsreduktion/Muskeltonustraining (z.B. Didgeridoo), RLS-Therapie (z.B. Eisensubstitution, Dopaminagonisten), Hypnotika? (Langzeittherapie nicht empfohlen!)

Abbildung 2. Wechselbeziehungen in der Schlafmedizin: Einige Schritte der Abklärung und Behandlung (die Wechselbeziehungen sind mannigfaltig und eine spezifische Diagnostik/Zuweisung ist oft notwendig). 
rung bei Risikogruppen mit möglicher Eigen- oder Fremdgefährdung (z.B. Berufskraftfahrer, beim Führen von Maschinen).

\section{Elektronisches Supplement}

Das elektronische Supplement (ESM) ist mit der OnlineVersion dieses Artikels verfügbar unter https://doi.org/ 10.1024/1661-8157/a003753.

ESM 1. Begriffsdefinitionen

ESM 2. Schlaf-Anamnese-Fragebogen (adaptiert nach R. Fiechter)

\section{Bibliografie}

1. Pollmächer T, Wetter T-C, Bassetti CLA, Högl B, Randerath W, Wiater A. Handbuch Schlafmedizin (German Edition). Amsterdam; Elsevier: 2020. Kindle-Version.

2. Heinzer R, Vat S, Marques-Vidal P, et al. Prevalence of sleepdisordered breathing in the general population: the HypnoLaus Study. Lancet Respir Med. 2015;3:310-318.

3. Fiechter, R: ESM 1: Beispiel einer Schlafanamnese, als Exceldokument.

4. Deutsche Gesellschaft für Schlafforschung und Schlafmedizin [DGSM] (Hg.). S3-Leitlinie Nicht erholsamer Schlaf/Schlafstörungen - Kapitel «Schlafbezogene Atmungsstörungen» Somnologie 2017.20 (Suppls2): S97-S180. DOI 10.1007/s11818016-0093-1. Publiziert: 29.11.2016

5. Mathis J, Kohler M, Hemmeter U-M, Seeger R. Fahreignung bei Tagesschläfrigkeit. Swiss Medical Forum. 2017;17:442-444. Verkehrskommission Schweizerische Gesellschaft für Schlafforschung, Schlafmedizin und Chronobiologie (SGSSC). www. swisssleep.ch/driving.

6. DEGAM-Anwenderversion zur S3 Leitlinie «Nicht erholsamer Schlaf/Schlafstörungen». Baum, Riemann. Stand 05/2017.

7. Falloon K, Elley CR, Fernando A, Lee AC, Arroll B. Simplified sleep restriction for insomnia in general practice: a randomised controlled trial. Br J Gen Pract. 2015;65(637): e508e515. DOI https://doi.org/10.3399/bjgp15X686137

8. American Academy of Sleep Medicine. International Classification of Sleep Disorders. 3rd ed. Darien, IL: American Academy of Sleep Medicine; 2014.

9. Mathis J. Langzeittherapie beim Restless-Legs Syndrom, Revue Méd Suisse. www.revmed.ch. 28.1.2015.
10. Dietmann A, Maire M, Bargiotas P, Mathis J, Auerb R, Bassetti $\mathrm{CL}$. Tagesschläfrigkeit, Hypersomnie oder Tagesmüdigkeit? Swiss Med For. 2019;19(19-20):319-324.

11. Hautzinger M, Keller F, Kühner C. Beck Depressions-Inventar (BDI-II). Revision. Frankfurt/Main: Hartcourt Test Services. 2006.

12. Johns MW. A new method for measuring daytime sleepiness. The Epworth Sleepiness Scale. Sleep. 1991;14:103-109.

13. Valko PO, Bassetti CL, Bloch KE, Held U, Baumann CR. Validation of the fatigue severity scale in a Swiss cohort. Sleep. 2008;31:1601-1607.

14. Bastien $\mathrm{CH}$, Vallieres A, Morin CM. Validation of the Insomnia Severity Index as an outcome measure for insomnia research. Sleep Med. 2001;2:297-307.

15. Horne JA, Ostberg O. A self-assessment questionnaire to determine morningness-eveningness in human circadian rhythms. Int J Chronobiol. 1976;(2):97-110. PMID: 1027738.

16. Marti-Soler, Hirotsu C, Marques-Vidal P, et al. The NoSAS score for screening of sleep-disordered breathing: a derivation and validation study. Lancet Respir Med. 2016;4:742-748

17. Buysse DJ, Reynolds CF 3rd, Monk TH, Berman SR, Kupfer DJ. The Pittsburgh Sleep Quality Index: a new instrument for psychiatric practice and research. Psychiatry Res. 1989;28:193213):

18. Crönlein T, et al. Regensburg Insomnia Scale (RIS): a new short rating scale for the assessment of psychological symptoms and sleep in insomnia; Study design: development and validation of a new short self-rating scale in a sample of 218 patients suffering from insomnia and 94 healthy controls. Health Qual Life Outcomes. 2013;11:65.

19. Chung F, Yegneswaran B, Liao P, et al. STOP questionnaire: A tool to screen patients for obstructive sleep apnea. Anesthesiology. 2008;108(5):812-821. DOI: 10.1097/ALN.0b013e31816d83e4.

20. Chung F, Abdullah HR, Liao P. STOP-Bang Questionnaire: A Practical Approach to Screen for Obstructive Sleep Apnea. Chest. 2016;149(3):631-638. DOI: 10.1378/chest.15-0903.

\section{Historie}

Manuskript eingereicht: 24.02.2021

Nach Revision angenommen: 27.07.2021

\section{Interessenskonflikte}

Es bestehen keine Interessenskonflikte.

\section{Patrick Heeb, dipl. Arzt}

Lungenfachzentrum Fiechter

Zürichstrasse 17

8607 Aathal

patrick.heeb@hin.ch 